\title{
Higher-order generalized uncertainty principle corrections to the Jeans mass
}

\author{
Zhong-Wen Feng ${ }^{1, \mathrm{a}}{ }_{\mathbb{O}}$, Guansheng $\mathrm{He}^{2}$, Xia Zhou ${ }^{1}$, Xue-Ling $\mathrm{Mu}^{3}$, Shi-Qi Zhou ${ }^{1}$ \\ ${ }^{1}$ Physics and Astronomy College, China West Normal University, Nanchong 637009, China \\ ${ }^{2}$ School of Mathematics and Physics, University of South China, Hengyang 421001, China \\ ${ }^{3}$ School of Information Science and Engineering, Chengdu University, Chengdu 610106, China
}

Received: 10 November 2020 / Accepted: 16 August 2021 / Published online: 21 August 2021

(C) The Author(s) 2021

\begin{abstract}
The Jeans instability is regarded as an important tool for analyzing the dynamics of a self-gravitating system. However, this theory is challenging since astronomical observation data show some Bok globules, whose masses are less than the Jeans mass and still have stars or at least undergo the star formation process. To explain this problem, we investigate the effects of the higher-order generalized uncertainty principle on the Jeans mass of the collapsing molecular cloud. The results in this paper show that the higher order generalized uncertainty principle has a very significant effect on the canonical energy and gravitational potential of idea gas, and finally leads to a modified Jeans mass lower than the original case, which is conducive to the generation of stars in small mass Bok globules. Furthermore, we estimate the new generalized uncertainty principle parameter $\gamma_{0}$ by applying various data of Bok globules, and find that the range of magnitude of $\gamma_{0}$ is $10^{11}-10^{12}$.
\end{abstract}

\section{Introduction}

Einstein's theory of general relativity is regarded as the cornerstone of the development of modern physics and astronomy. However, with the deepening of research, it is found that general relativity has flaws, which lead to many problems, such as the black hole information paradox and naked singularity of spacetimes [1]. One of the most promising candidates to solve those problems is quantum gravity $(\mathrm{QG})$. Now, based on these frameworks that explicate QG, people hypothesize that exists a minimum measurable length of the order of the Planck length in QG, which is supported by Gedanken experiments [2-4].

According to the minimum measurable length, many such studies have converged on the idea that the Heisenberg

a e-mail: zwfengphy@cwnu.edu.cn (corresponding author) uncertainty principle (HUP) can be modified as the generalized uncertainty principle (GUP). In 1995, Kempf, Mangano and Mann proposed a quadratic form of the GUP, which we now call as the "KMM model" with the expression, $\Delta x \Delta p \geq \frac{\hbar}{2}\left[1+\beta \Delta p^{2}\right]$, where $\beta=\beta_{0} / M_{p}^{2} c^{2}=\beta_{0} \ell_{p}^{2} / \hbar^{2}$ and $\beta_{0}$ is the GUP parameter. It is easy to find that the KMM model predicts a minimal length $\Delta x_{\min }^{\mathrm{KMM}} \approx \ell_{p} \sqrt{\beta_{0}}$ [5]. Subsequently, incorporating the idea of maximal momentum, Ali, Das and Vagenas constructed another GUP (ADV model) $\Delta x \Delta p \geq \frac{\hbar}{2}\left[1+2 \alpha_{0} \ell_{p}\langle p\rangle / \hbar+4\left\langle p^{2}\right\rangle \alpha_{0}^{2} \ell_{p}^{2} / \hbar^{2}\right]$, where $\alpha_{0}$ is the GUP parameter. Moreover, this linear and quadratic GUP model suggests the existence of a minimal length $\Delta x_{\min }^{\mathrm{ADV}} \approx \alpha_{0} \ell_{p}$ and a maximal momentum $\Delta p_{\max }^{\mathrm{ADV}} \approx \ell_{p} / \alpha_{0}$ [6]. In recent years, the KMM model and ADV model have played important roles in the research of many physics contexts. For instance, in Refs. [7,8], the Scardigli et al., discussed the relation between GUP, general relativity, and the Lorentz violation. Besides, by using the KMM model, researchers derived the GUP corrected Hamilton-Jacobi equation and investigated the modified tunneling rate of particles with arbitrary spins from the event of curved spacetimes [9]. Moreover, the KMM model and ADV model can also be extended to the Proca equation, which leads to the modified Hawking temperature of black holes [10-12]. In addition, Vagenas et al. studied the validity of the no-cloning theorem within the framework of GUP. They pointed out that the energy required to send information to a black hole is affected by quantum gravity [13]. In Refs. $[14,15]$, the authors investigated how the KMM model affects the Casimir wormhole spacetime, and obtained a class of asymptotically flat wormhole solutions.

Although the KMM model and ADV model are two of the most studied GUPs, they still have some defects, e.g., the minimal length and the maximal momentum are only valid for small GUP parameters, and do not imply noncommutative 
geometry [16]. To overcome these difficulties, Pedram introduced a nonperturbative higher-order GUP, which agrees with various proposals of QG [17]. Subsequently, this higher order GUP was used to correct the blackbody radiation spectrum and predict the cosmological constant. Based on this heuristic work, many new forms of higher order GUP were presented (see, e.g. Refs. [18-21] and references therein). Recently, in Ref. [18], Chung and Hassanabadi proposed a generalized canonical commutation relations as follows:

$[\hat{x}, \hat{p}]=\frac{i \hbar}{1-\gamma|\hat{p}|}, \quad \gamma>0$

which leads to a new higher order GUP (hereafter, we call this GUP as the $\mathrm{CH}$ model):

$$
\begin{aligned}
\Delta x & \Delta p \geq \frac{\hbar}{2}\left\langle\frac{1}{1-\gamma|p|}\right\rangle \\
= & \frac{\hbar}{2}\left\langle 1+\gamma|p|+\gamma^{2} P^{2}+\gamma^{3}|p| p^{2}+\gamma^{4}\left(p^{2}\right)^{2}+\cdots\right\rangle \\
\geq & \frac{\hbar}{2}\left[1+\gamma\langle|p|\rangle+\gamma^{2}(\Delta p)^{2}+\gamma^{3}(\Delta p)^{3}\right. \\
& \left.\left.+\gamma^{4}(\Delta p)^{4}+\cdots\right\rangle\right] \\
\geq & \frac{\hbar}{2}\left[-\gamma(\Delta p)+1+\gamma(\Delta p)+\gamma^{2}(\Delta p)^{2}+\gamma^{3}(\Delta p)^{3}\right. \\
& \left.\left.+\gamma^{4}(\Delta p)^{4}+\cdots\right\rangle\right]=\frac{\hbar}{2}\left[-\gamma(\Delta p)+\frac{1}{1-\gamma(\Delta p)}\right],
\end{aligned}
$$

where $|p|=\sqrt{\left|p^{2}\right|}$ and $\gamma=\gamma_{0} / M_{p} c=\gamma_{0} \ell_{p} / \hbar$ are associated with the dimensionless GUP parameter $\gamma_{0}$, the Planck mass $M_{p}$, and the Planck length $\ell_{p}$. In addition, we set $\langle p\rangle=0,\langle|p|\rangle \geq 0$ and utilized the identities $\left\langle\left(p^{2}\right)^{n}\right\rangle \geq\left(\left\langle p^{2}\right\rangle\right)^{n}$ and $|\langle(A B+B A)\rangle| \geq 2 \sqrt{\left\langle A^{2}\right\rangle} \sqrt{\left\langle B^{2}\right\rangle}$ [18]. In addition, Eq. (2) guarantees the existence of an absolute smallest uncertainty in position $\Delta x_{\min }=3 \hbar \gamma / 2$ for $\Delta p=1 / 2 \gamma$, which never appears in the framework of the HUP. In addition, the $\mathrm{CH}$ model has merit since it involves no perturbation and overcomes some conceptual problems raised in the previous forms of GUP (e.g. the divergence of the energy spectrum of the eigenfunctions of the position operator), however, when $\gamma \rightarrow 0$, it reduces to the HUP. Besides, for $\gamma \ll 1$, the last term of Eq. (2) can be expanded as $\Delta x \Delta p \geq \frac{\hbar}{2}\left[1+\gamma^{2} \Delta p^{2}+\mathcal{O}\left(\gamma^{3}\right)\right]$, which indicates that the $\mathrm{CH}$ model coincides with the KMM model to the second order in $\gamma$; hence, one has the relation $\gamma^{2} \sim \beta$.

According to the $\mathrm{CH}$ model (2), people investigated the modified eigenfunctions and eigenvalues for the particle in a box and one-dimensional hydrogen atom, respectively. Moreover, one may also find that the higher order GUP corrected the behavior of Bohr-Sommerfeld quantization, which can be used to calculate the energy spectra of quantum harmonic oscillators and quantum bouncers [20]. In light of previous works, it is believed that a higher-order GUP will not only give new implications to quantum systems, but also to astrophysics. For example, it is well known that the Jeans mass is an important theory to study the collapse of molecular clouds. However, in recent years, this theory is has been since some astronomical data have shown that there are some Bok globules, whose masses are less than the Jeans mass that still have stars or at least undergo the star formation process $[22,23]$. For solving this problem, Moradpour et al. corrected the limit of Jeans mass due to the KMM model. Their results showed that the modifications were less than the original case and were related to the properties of the GUP model [24]. As we discussed above, the higher order GUP has different properties from the KMM model and the ADV model, and it is interesting to investigate how the higher order GUP affects the Jeans mass. Therefore, in the present paper, we try to extend the $\mathrm{CH}$ model (2) into the Jeans instability and calculate the GUP corrected limit of Jeans mass of those especial Bok globules.

The paper is organized as follows: Sect. 2 is devoted to a review of the Jeans instability limit in the classical case. In Sect. 3, according to the new higher order $\mathrm{CH}$ model, we compute the corrections to the potential energy and canonical energy of a molecular cloud. Then, we discuss the modified Jeans gravitational instability, and then derive the GUP corrected Jeans mass to explain the problem of Bok globules. By using the GUP corrected Jeans mass, the dimensionless parameter $\gamma_{0}$ of the GUP is constrained in Sect. 4. Finally, conclusions can be found in Sect. 5 .

\section{The Jeans mass and the HUP}

In this section, we briefly outline how to derive the Jeans mass in the classical case. Based on the argument that relies upon the virial theorem in Ref. [24], the collapse of the molecular cloud occurs if the gravitational potential energy $E_{p}$ is larger than the canonical energy $U$, to wit

$U<-E_{p} / 2$

Obviously, to obtain the Jeans mass, it is necessary to derive the expressions of gravitational potential energy and canonical energy.

It is well known that spacetime is flat in the classical case. Therefore, the Newton's law of gravitation is $F_{0}=$ $G M m / r^{2}$ with the constant of universal gravitation $G$, and the gravitational potential becomes $V(r)=-G M / r$. The corresponding potential energy is given by 


$$
\begin{aligned}
E_{p} & =\int_{0}^{M} V(r) d r=\int_{0}^{R} 4 \pi \rho(r) r G M d r \\
& =-\frac{3 G M^{2}}{5 R}
\end{aligned}
$$

where the density of dark cloud $\rho(r)$ in Eq. (4) is assumed to be a constant $\rho_{0}$ for simplicity.

Next, the classical fundamental commutation relation can be expressed as $[\hat{x}, \hat{p}]=i \hbar$, which implies HUP $\Delta x \Delta p \geq$ $\hbar / 2$. When considering the Liouville theorem and HUP, the state density in momentum space in the spherical coordinate system can be expressed as $D(p) d p=4 \pi V p^{2} d p / h^{3}$, which leads directly to the original partition function of the ideal gas as follows

$$
\begin{aligned}
Z & =\frac{\mathcal{Z}^{N}}{N !}=\frac{(4 \pi V)^{N}}{N !} \int\left[\frac{p^{2}}{h^{3}} \exp \left(-\frac{p^{2}}{2 \mu k_{B} T}\right)\right]^{N} d p \\
& =\frac{(4 \pi V)^{N}}{N !} \frac{\left(2 \pi \mu k_{B} T\right)^{3 N / 2}}{h^{3 N}},
\end{aligned}
$$

where $N, \mu$, and $T$ are the numbers, mass, and temperature of noninteracting particles of the ideal gas, respectively [25-27]. Besides, the total mass of the ideal gas satisfies the relationship $M=\mu N$. According to Eq. (5), one can straightforward to derive the equation of state of the ideal gas $P V=N k_{B} T$, and the canonical energy can be expressed as

$U_{0}=k_{B} T^{2}\left(\frac{\partial \ln Z}{\partial T}\right)_{N, V}=\frac{3}{2} N k_{B} T$.

Clearly, the original canonical energy is dependent only on the number of noninteracting particles $N$ and their temperature $T$.

Now, substituting Eq. (4) and Eq. (6) into inequality (3), and supposing that the distribution of ideal gas in space is spherical, the result is

$M>\left(\frac{5 k_{B} T}{G \mu}\right)^{\frac{3}{2}}\left(\frac{3}{4 \pi \rho_{0}}\right)^{\frac{1}{2}}$,

where the radius is $R=\left(3 M / 4 \pi \rho_{0}\right)^{\frac{1}{3}}$. The above mentioned equation indicates a lower bound of the cloud mass to collapse, which is known as the Jeans mass

$M_{0}^{J}=\left(\frac{5 k_{B} T}{G \mu}\right)^{\frac{3}{2}}\left(\frac{3}{4 \pi \rho_{0}}\right)^{\frac{1}{2}}$.

Although the formation of most stars can be examined by the original Jeans instability and Jeans mass, people still observed some Bok globules, such as CB 84 and CB 110, have masses less than $M_{0}^{J}$ but still have stars or at least undergo the star formation process [23]. To explain this problem, we investigate the modified Jeans mass in the framework of the higher-order GUP.

\section{The Jeans mass in the higher-order GUP}

The GUP has various implications for a wide range of physical systems. Moreover, they are regarded as a powerful tool to solve various difficult problems in these research fields. Therefore, to obtain the modified Jeans mass, we derive the GUP corrected entropy, gravitation potential energy, and canonical energy of ideal gases. For the sake of simplicity, we shall takes the units $\hbar=c=k_{B}=1$ in the next research.

\subsection{GUP corrected gravitation potential energy}

To derive the modified gravitation potential energy in the presence of the $\mathrm{CH}$ model, we need to use the theory of entropic force, which is an intriguing explanation for Newton's law of gravity that is based on the holographic principle and an equipartition rule. Now, by using Eq. (2), one can easily obtain the following inequality

$$
\begin{aligned}
\Delta p & \geq \frac{1}{2 \gamma}\left(1-\sqrt{1-\frac{4 \gamma}{2 \Delta x+\gamma}}\right) \\
& =\frac{1}{2 \Delta x}\left[1+\frac{\gamma^{2}}{4 \Delta x^{2}}+\mathcal{O}\left(\gamma^{3}\right)\right] .
\end{aligned}
$$

Based on the arguments of Refs. [29-33], a massless quantum particle (e.g., a photon) can be used to determine the position of quantum particles with the energy $\omega=p c$ (i.e. $\Delta \omega=$ $\Delta p c$ ), so that the HUP $\Delta p \geq 1 / 2 \Delta x$ can be translated to the lower bound $\omega \geq 1 / 2 \Delta x$. Accordingly, from Eq. (9), the GUP version reads

$\omega \geq \frac{1}{2 \Delta x}\left[1+\frac{\gamma^{2}}{4 \Delta x^{2}}+\mathcal{O}\left(\gamma^{3}\right)\right]$.

From Verlinde's entropy force theory, to calculate the modified entropic force, one should consider a spherically symmetric gravitational system (e.g., a black hole), which allows the quantum particles to enter or exit its horizon [34]. As Ref. [35] pointed out, when a strong gravitational system absorbs or emits a quantum particle with energy $\omega$ and size $\mathcal{R}$, the minimal change in the area of the system becomes $\Delta A_{\min } \geq 8 \pi \omega \mathcal{R} \ell_{p}^{2}$. Then, the arguments of Refs. [36,37] implies that the size of a quantum particle cannot be smaller than its uncertainty in the position (i.e. $\mathcal{R} \geq \Delta x$ ), which gives $\Delta A_{\min } \geq 8 \pi \omega \Delta x \ell_{p}^{2}$. Substituting this relation into Eq. (10), one has 
$\Delta A_{\min } \geq 4 \pi \ell_{p}^{2}\left[1+\frac{\gamma^{2}}{4 \Delta x^{2}}+\mathcal{O}\left(\gamma^{3}\right)\right]$.

Furthermore, considering that a spherical gravitational system with Schwarzschild radius $r$, and the area of this system is $A=4 \pi r^{2}$. Moreover, near the event horizon, $\Delta x$ is approximately equal to the orbital radius $r$; that is, $\Delta x \approx 2 r[32,38-$ 40]. Hence, the relation between $\Delta x$ and $A$ is given by $\Delta x^{2}=4 r^{2}=A / \pi$. Substituting this relation into Eq. (11), the minimal change in the horizon area of the gravitational system can be recast as

$\Delta A_{\min } \simeq \lambda \ell_{p}^{2}\left[1+\frac{\pi \gamma^{2}}{4 A}+\mathcal{O}\left(\gamma^{3}\right)\right]$,

where $\lambda$ is an undetermined coefficient. In Ref. [36], the authors pointed out that the information of the gravitational system is reflected in its area. On the other hand, based on the information theory, it is believed that the area of one system can affect its smallest increase in entropy [41]. Since the fundamental unit of entropy as one bit of information is $\Delta S_{\min }=b=\ln 2$, one obtains

$\frac{\mathrm{d} S}{\mathrm{~d} A}=\frac{\Delta S_{\min }}{\Delta A_{\min }}=\frac{b}{\lambda \ell_{p}^{2}}\left[1+\frac{\pi \gamma^{2}}{4 A}+\mathcal{O}\left(\gamma^{3}\right)\right]^{-1}$,

Considering that $\gamma$ is a small parameter, one can obtain the GUP corrected entropy according to Eq. (13),

$S=\frac{A}{4 \ell_{p}^{2}}\left[1-\frac{\pi \gamma^{2}}{4 A} \ln (4 A)+\mathcal{O}\left(\gamma^{3}\right)\right]$,

where we fix $b / \lambda$ by demanding matching the original entropy-area law in the limit $\gamma \rightarrow 0$. Hence, one has $b / \lambda=1 / 4$ [35]. Moreover, there is a logarithmic term $\ln (4 A)$ in the bracketed term of Eq. (14), which is coincident with the requirements of QG [42-49]. According to the holographic principle and entropy-area law, which is averagely distributed in $\mathcal{N}$-bits information, the number of bits can be expressed as follows:

$\mathcal{N}=4 S=\frac{A}{\ell_{p}^{2}}\left[1-\frac{\pi \gamma^{2}}{4 A} \ln (4 A)+\mathcal{O}\left(\gamma^{3}\right)\right]$.

Next, one can further denote the total energy of the gravitational system as $E$, which is the average distribution in $\mathcal{N}$ bits (please see the [50] for details). Consequently, each bit contains $T / 2$ energy, then, following the equipartition rule, the total energy takes the form

$E=\mathcal{N} T / 2$.
On the other hand, Verlinde demonstrated that the entropic force is more fundamental than gravity. For a gravitational system, the entropic force can be expressed as follows [34]:

$F \Delta x=T \Delta S$

where $F$ is the entropy force, $T$ is the temperature, $\Delta S$ is the change in entropy of the gravitational system, and $\Delta x$ represents the displacement of the particle with the mass $m$ from the gravitational system, which satisfies the relation $\Delta S=2 \pi m \Delta x[51,52]$. Now, substituting the expression of the change in entropy, Eq. (14)-Eq. (16) into Eq. (17), then considering $E=M c^{2}, A=4 \pi r^{2}$ and $\ell_{p}^{2}=G$, Newton's law of gravitation should be corrected as follows:

$F_{\mathrm{GUP}}=\frac{G M m}{r^{2}}\left[1+\frac{\gamma^{2}}{16 r^{2}} \ln \left(16 \pi r^{2}\right)+\mathcal{O}\left(\gamma^{3}\right)\right]$.

Clearly, in the limit $\gamma=0$, Eq. (18) reduce to the original Newton's gravitation $F_{0}=G M m / r^{2}$. Next, based on the modified Newton's law of gravitation, the corresponding GUP corrected gravitational potential is

$$
\begin{aligned}
V_{\mathrm{GUP}} & =\int \frac{F_{\mathrm{GUP}}}{m} d r \\
& =-\frac{G M}{r}\left[1+\frac{2+3 \ln \left(16 \pi r^{2}\right)}{144 r^{2}} \gamma^{2}+\mathcal{O}\left(\gamma^{3}\right)\right] .
\end{aligned}
$$

Applying Eq. (19) to a molecular cloud with radius $R$, mass $M$ and the almost uniform density $\rho_{0}$, the following expression for the modified potential energy is obtained

$$
\begin{aligned}
E_{p}^{\mathrm{GUP}} & =\int_{0}^{R} V_{\mathrm{GUP}}(r) d M \\
& =-\frac{3 G M^{2}}{5 R}\left[1+\frac{5 \ln \left(16 \pi R^{2}\right)}{144 R^{2}} \gamma^{2}+\mathcal{O}\left(\gamma^{3}\right)\right] .
\end{aligned}
$$

Obviously, the above equation is related to the mass $M$, radius $R$, and GUP parameter $\gamma$. In addition, one can see that Eq. (20) lacks the first-order correction term since the $\mathrm{CH}$ model (2) becomes the KMM model for small $\gamma$. This indicates that the properties of the gravitational system are affected by the higher-order term of $\gamma$. In the limit $\gamma=0$, the result agrees with the original potential energy (4).

\subsection{GUP corrected canonical energy of ideal gases}

By virtue of Eq. (2) and the viewpoint in Refs. [53,54], the partition function of a dark cloud, which is approximated as 
the ideal gas containing $N$ noninteracting particles with mass $m$ at temperature $T$, can be expressed as follows:

$Z=\frac{\mathcal{Z}^{N}}{N !}$,

where

$\mathcal{Z}=\frac{4 \pi V}{N !} \int_{0}^{\infty} \frac{p^{2}}{h^{3}}(1-3 \gamma|p|)^{3} \exp \left(-\frac{p^{2}}{2 \mu T}\right) d p$.

Using the spherical coordinate and Gaussian integral, the GUP corrected partition function for the dark cloud reads

$Z_{\mathrm{GUP}}=\frac{(4 \pi V)^{N}\left(2 k_{B} \mu T\right)^{3 N / 2}}{N ! h^{3 N}} \Theta^{N}(\gamma)$,

where $\Theta(\gamma)=\frac{\sqrt{\pi}}{4}\left[1-6 \sqrt{\frac{2 \mu T}{\pi}} \gamma+9 \mu T \gamma^{2}+\mathcal{O}\left(\gamma^{3}\right)\right]$. The GUP corrected canonical energy is presented as

$$
\begin{aligned}
U_{\mathrm{GUP}} & =T^{2}\left(\frac{\partial \ln Z_{\mathrm{GUP}}}{\partial T}\right)_{N, V} \\
& =U_{0}\left[1-2 \sqrt{\frac{2}{\pi}} \mathcal{A} \gamma-\frac{6}{\pi}(4-\pi) \mathcal{A}^{2} \gamma^{2}+\mathcal{O}\left(\gamma^{3}\right)\right]
\end{aligned}
$$

with $\mathcal{A}=\sqrt{\mu T}$ and the original canonical energy $U_{0}$ is given in Eq. (6). Note that, the modified canonical energy is different from the original case, it does only not relate to the original case $U_{0}$, but also to parameter $\gamma$, the mass of the noninteracting particle $\mu$ and its temperature $T$.

\subsection{GUP corrected Jeans mass}

With the above results in place, one can derive the modified Jeans mass and further investigate how the GUP affects the Jeans mass. Using Eq. (20) and Eq. (24) and recalling Eq. (3), one yields

$$
\begin{gathered}
N T\left[1-2 \sqrt{\frac{2}{\pi}} \mathcal{A} \gamma-\frac{6}{\pi}(4-\pi) \mathcal{A}^{2} \gamma^{2}+\mathcal{O}\left(\gamma^{3}\right)\right] \\
<\frac{G M^{2}}{5 R}\left[1+\frac{5 \ln \left(16 \pi R^{2}\right)}{144 R^{2}} \gamma^{2}+\mathcal{O}\left(\gamma^{3}\right)\right] .
\end{gathered}
$$

Considering the radius $R=\left(3 M / 4 \pi \rho_{0}\right)^{\frac{1}{3}}$, Eq. (25) can be rewritten as follows:

$$
\frac{5 T\left[1-2 \sqrt{\frac{2}{\pi}} \mathcal{A} \gamma-\frac{6}{\pi}(4-\pi) \mathcal{A}^{2} \gamma^{2}+\mathcal{O}\left(\gamma^{3}\right)\right]}{G \mu\left(4 \pi \rho_{0} / 3\right)^{\frac{1}{3}}}<M^{\frac{2}{3}} \chi,
$$

where $\chi=1+\frac{5 \gamma^{2}}{144\left(3 M / 4 \pi \rho_{0}\right)^{2}} \ln \left[16 \pi\left(3 M / 4 \pi \rho_{0}\right)^{2}\right]+\mathcal{O}$ $\left(\gamma^{3}\right)$. On the one hand, if $\gamma=0$, the upper bound of mass goes to the Jeans mass $M_{0}^{J}=(5 T / G \mu)^{\frac{3}{2}}\left(3 / 4 \pi \rho_{0}\right)^{\frac{1}{2}}$. On the other hand, because $M \gg 1$ and $\gamma$ is finite, the GUP corrected Jeans mass is obtained by saturating Eq. (26), with the result

$$
\begin{aligned}
& M_{\mathrm{GUP}}^{J}=M \\
& \quad>M_{0}^{J}\left[1-\sqrt{\frac{8 \mu T}{\pi}} \gamma-(4-\pi) \frac{6 \mu T}{\pi} \gamma^{2}+\mathcal{O}\left(\gamma^{3}\right)\right]^{\frac{3}{2}} .
\end{aligned}
$$

One may see that the GUP corrected Jeans mass $M_{\text {GUP }}^{J}$ is related to the original Jeans mass $M_{0}^{J}$, the mass of ideal gas $\mu$, the temperature of gravity system $T$, and the GUP parameter $\gamma$. Furthermore, it should be noted that the mass of ideal gas and the temperature of the gravity system must be real numbers greater than zero. When considering $\gamma>0, M_{\mathrm{GUP}}^{J}$ is positive when $1>\sqrt{8 \mu T / \pi} \gamma-(4-\pi) 6 \mu T \gamma^{2} / \pi+$ $\mathcal{O}\left(\gamma^{3}\right)$ is lower than $M_{0}^{J}$. To date, the astronomical observations have shown that some Bok globules' masses are less than their corresponding Jeans masses. The GUP corrections to the Jeans mass can be a candidate to explain those observational facts. Our results show that the effect of GUP is able to reduce the Jeans mass, which is conducive to the generation of stars in small-mass Bok globules.

\section{Constraints for GUP parameter $\gamma_{0}$}

The GUP parameters are always assumed to be of order unity so that the modified results are negligible unless energy approaches the Planck scale. However, if the assumptions regarding the GUP parameters are not considered, the bound of GUP parameters can be obtained by previous experimental and observational data. In previous works, people mainly focused on the constraints on the GUP parameters of the KMM model and the ADD model [55-71]. Now, armed with the previous results, we estimate the parameter of the $\mathrm{CH}$ model based on the data of Bok globules. First, for simplicity, by keeping the leading order term of $\gamma$, inequality (27) can be written as follows

$M_{\mathrm{GUP}}^{J}>M_{0}^{J}\left(1-\sqrt{\frac{8 \mu T}{\pi}} \frac{\gamma_{0}}{M_{p} c}\right)^{\frac{3}{2}}$. 
Table 1 The ID, temperature $T$, mass $M_{\mathrm{GUP}}^{J}$ and Jeans mass $M_{0}^{J}$ of Bok globules, $\gamma_{0}$ and $\gamma_{0}^{2}$ are the first power and second power of the upper bound on the GUP parameter of the $\mathrm{CH}$ model, $\beta_{0}^{\mathrm{KMM}}$ is the upper bound on the GUP parameter of the KMM model

\begin{tabular}{|c|c|c|c|c|c|c|}
\hline ID & $\begin{array}{c}T \\
(\mathrm{~K})\end{array}$ & $\begin{array}{l}M_{\mathrm{GUP}}^{J}[22] \\
\left(\mathrm{M}_{\odot}\right)\end{array}$ & $\begin{array}{c}M_{0}^{J} \\
\left(\mathrm{M}_{\odot}\right)\end{array}$ & $\gamma_{0}$ & $\gamma_{0}^{2}$ & $\beta_{0}^{\mathrm{KMM}}[24]$ \\
\hline CB 87 & 11.4 & 2.73 & 9.6 & $5.31 \times 10^{12}$ & $2.82 \times 10^{24}$ & $6.33 \times 10^{25}$ \\
\hline CB 110 & 21.8 & 7.21 & 8.5 & $7.03 \times 10^{11}$ & $5.00 \times 10^{23}$ & $6.11 \times 10^{24}$ \\
\hline CB 131 & 25.1 & 7.83 & 8.1 & $1.41 \times 10^{11}$ & $1.99 \times 10^{22}$ & $1.02 \times 10^{24}$ \\
\hline CB 161 & 12.5 & 2.79 & 5.4 & $3.18 \times 10^{12}$ & $1.01 \times 10^{25}$ & $3.62 \times 10^{26}$ \\
\hline CB 188 & 19.0 & 7.19 & 7.7 & $3.24 \times 10^{11}$ & $1.05 \times 10^{23}$ & $3.05 \times 10^{24}$ \\
\hline FeSt 1-457 & 10.9 & 1.12 & 1.4 & $1.32 \times 10^{12}$ & $1.75 \times 10^{24}$ & $1.60 \times 10^{25}$ \\
\hline Lynds 495 & 12.6 & 2.95 & 6.6 & $1.70 \times 10^{12}$ & $1.37 \times 10^{25}$ & $4.20 \times 10^{25}$ \\
\hline Lynds 498 & 11.0 & 1.42 & 5.7 & $5.76 \times 10^{12}$ & $3.31 \times 10^{25}$ & $6.99 \times 10^{25}$ \\
\hline
\end{tabular}

By solving the above equation, one yields

$\gamma_{0}<\frac{c M_{p}}{2} \sqrt{\frac{\pi}{2 \mu T}}\left[1-\left(\frac{M_{\mathrm{GUP}}^{J}}{M_{0}^{J}}\right)^{2 / 3}\right]$.

Now, based on the data of Bok globules in Ref. [22], the upper bounds on $\gamma_{0}$ are shown in Table 1 .

In Table 1 , we set $M_{p}=2.18 \times 10^{-8} \mathrm{~kg}, c=2.99 \times$ $10^{8} \mathrm{~m} \cdot \mathrm{s}^{-1}$, and $\mathrm{M}_{\odot}$ denotes the Sun mass. The final results are determined by the mass of noninteracting particles $\mu$. To obtain the exact value of the upper bounds on $\gamma_{0}$, we further assume that the noninteracting particles are composed of hydrogen atoms, which are the most abundant element in the universe with the mass $\mu_{\mathrm{H}}=1.67 \times 10^{-26} \mathrm{~kg}$. In this case, one can see that the range of upper bounds of $\gamma_{0}$ is $10^{11} \sim 10^{12}$. Furthermore, the GUP parameter in the $\mathrm{CH}$ model has a special relationship with that of the KMM model, which can be inferred from Eq. (2), as $\gamma^{2} \sim \beta$ (or $\gamma_{0}^{2} \sim \beta_{0}$ ). Hence, it is easy to find that the upper bound of the GUP parameter in the $\mathrm{CH}$ model $\gamma_{0}^{2}$ is $1-2$ orders of magnitude more stringent than those in the KMM model $\beta_{0}^{\mathrm{KMM}}<\left[1-\left(M_{G U P}^{J} / M_{0}^{J}\right)^{2 / 3}\right] M_{p}^{2} c^{2} / 2 \mu T$ from Ref. [24].

\section{Discussion}

In this paper, by incorporating a new higher-order GUP with virial equilibrium and Verlinde's entropy force theory, we investigated the modified canonical energy $U_{\mathrm{GUP}}$ and the modified gravitational potential energy $E_{p}^{\mathrm{GUP}}$ of a molecular cloud. Subsequently, according to those modifications, the GUP corrected Jeans mass $M_{\mathrm{GUP}}^{J}$ is obtained. It is found that the GUP can effectively increase the gravitational potential, and reduce the canonical energy and the Jeans mass. This leads to the collapse of Bok globules with masses less than the standard value $M_{0}^{J}$, which is consistent with astronomical observations. Finally, using the different data of Bok globules, we constrain the upper bounds of the GUP parameter $\gamma_{0}$, whose range turn out to be $10^{11} \sim 10^{12}$.

Acknowledgements The authors thank the anonymous referees for helpful suggestions and enlightening comments, which helped to improve the quality of this paper. This work is supported in part by the National Natural Science Foundation of China (Grant Nos. 12105231 and 11947018) and the Fundamental Research Funds of China West Normal University (Grant Nos. 17E093 and 18Q067).

Data Availability Statement This manuscript has no associated data or the data will not be deposited. [Authors' comment: This is a theoretical study and no experimental data has been listed.]

Open Access This article is licensed under a Creative Commons Attribution 4.0 International License, which permits use, sharing, adaptation, distribution and reproduction in any medium or format, as long as you give appropriate credit to the original author(s) and the source, provide a link to the Creative Commons licence, and indicate if changes were made. The images or other third party material in this article are included in the article's Creative Commons licence, unless indicated otherwise in a credit line to the material. If material is not included in the article's Creative Commons licence and your intended use is not permitted by statutory regulation or exceeds the permitted use, you will need to obtain permission directly from the copyright holder. To view a copy of this licence, visit http://creativecomm ons.org/licenses/by/4.0/.

Funded by SCOAP 3 .

\section{References}

1. P. Chen, Y.C. Ong, D.-H. Yeom, Phys. Rep. 603, 1 (2015). arXiv: 1412.8366

2. M. Maggiore, Phys. Lett. B 319, 83 (1993). arXiv:hep-th/9309034

3. G. Amelino-Camelia, Int. J. Mod. Phys. D 11, 35 (2002). arXiv:gr-qc/0012051

4. F. Scardigli, Phys. Lett. B 452, 39 (1999). arXiv:hep-th/9904025

5. A. Kempf, G. Mangano, R.B. Mann, Phys. Rev. D 52, 1108 (1995). arXiv:hep-th/9412167

6. A.F. Ali, S. Das, E.C. Vagenas, Phys. Lett. B 678, 497 (2009). https://doi.org/10.1016/j.physletb.2009.06.061 
7. G. Lambiase, F. Scardigli, Phys. Rev. D 97, 075003 (2018). arXiv: 1709.00637

8. R. Casadio, F. Scardigli, Phys. Lett. B 807, 135558 (2020). arXiv:2004.04076

9. Z.W. Feng, H.L. Li, X.T. Zu, Tao, S.Z. Yang, Eur. Phys. J. C 76, 212 (2016). arXiv: 1604.04702

10. I. Sakalli, A. Övgün, Europhys. Lett. 118, 60006 (2017). arXiv: 1702.04636

11. A. Övgün, K. Jusufi, Eur. Phys. J. Plus 132, 298 (2017). arXiv: 1703.08073

12. F. Scardigli, M. Blasone, G. Luciano, R. Casadio, Eur. Phys. J. C 78, 728 (2018). https://doi.org/10.1140/epjc/s10052-018-6209-y

13. E.C. Vagenas, A.F. Ali, H. Alshal, Eur. Phys. J. C 79, 276 (2019). arXiv:1811.06614

14. K. Jusufi, P. Channuie, M. Jamil, Eur. Phys. J. C 80, 127 (2020). arXiv:2002.01341

15. M. Blasone, G. Lambiase, G. Luciano, L. Petruzziello, F. Scardigli, Int. J. Mod. Phys. D 29, 2050011 (2020). https://doi.org/10.1142/ S021827182050011X

16. P. Pedram, Phys. Lett. B 714, 317 (2012). arXiv:1110.2999

17. P. Pedram, Phys. Lett. B 718, 638 (2012). https://doi.org/10.1016/ j.physletb.2012.10.059

18. W.S. Chung, H. Hassanabadi, Eur. Phys. J. C 79, 213 (2019). https:// doi.org/10.1140/epjc/s10052-019-6718-3

19. H. Hassanabadi, E. Maghsoodi, W.S. Chung, Eur. Phys. J. C 79, 358 (2019). https://doi.org/10.1140/epjc/s10052-019-6871-8

20. H. Shababi, W.S. Chung, Mod. Phys. Lett. A 33, 1850068 (2018). https://doi.org/10.1142/S0217732320500182

21. H. Shababi, W.S. Chung, Phys. Lett. B 770, 445 (2017). https:// doi.org/10.1016/j.physletb.2017.05.015

22. J. Vainio, I. Vilja, Gen. Relativ. Gravit. 48, 129 (2016). arXiv: 1512.04220

23. R. Kandori, Y. Nakajima, M. Tamura, K. Tatematsu, Y. Aikawa, T. Naoi, K. Sugitani, H. Nakaya, T. Nagayama, T. Nagata, M. Kurita, D. Kato, C. Nagashima, S. Sato, Astron. J. 130, 2166 (2005). arXiv: astro-ph/0506205

24. H. Moradpour, A.H. Ziaie, S. Ghaffari, F. Feleppa, Mon. Not. R Astron. Soc. 488, L69 (2019). arXiv:1907.12940

25. L.N. Chang, D. Minic, N. Okamura, T. Takeuchi, Phys. Rev. D 65, 125028 (2002). arXiv:hep-th/0201017

26. P. Wang, H. Yang, X. Zhang, J. High Energy Phys. 1008, 043 (2010). arXiv:1006.5362

27. W.S. Chung, H. Hassanabadi, Int. J. Mod. Phys. A 34, 1950041 (2019). https://doi.org/10.1142/S0217751X19500416

28. Z.-W. Feng, S.-Z. Yang, H.-L. Li, X.-T. Zu, Adv. High Energy Phys. 2016, 2341879 (2016). arXiv:1607.04114

29. R.J. Adler, P. Chen, D.I. Santiago, Gen. Relativ. Gravit. 33, 2101 (2001). https://doi.org/10.1023/A:1015281430411

30. M. Cavaglia, S. Das, R. Maartens, Class. Quantum Gravity 20, L205 (2003). arXiv:hep-ph/0305223

31. A.J.M. Medved, E.C. Vagenas, Phys. Rev. D 70, 12402 (2004). arXiv:hep-th/0411022

32. G. Amelino-Camelia, M. Arzano, A. Procaccini, Phys. Rev. D 70, 107501 (2004). arXiv:gr-qc/0405084

33. I.H. Belfaqih, H. Maulana, A. Sulaksono, Int. J Mod. Phys. D. https://doi.org/10.1142/S0218271821500644

34. E. Verlinde, J. High Energy Phys. 2011, 29 (2011). arXiv: 1001.0785

35. A. Awad, A.F. Ali, Cent. Eur. J. Phys. 12, 245 (2014). arXiv: 1403.5319

36. J.D. Bekenstein, Phys. Rev. D 7, 2333 (1973). https://doi.org/10. 1103/PhysRevD.7.2333

37. A. Awad, A.F. Ali, J. High Energy Phys. 2014, 93 (2014). arXiv: 1404.7825

38. B. Majumder, Phys. Lett. B 703, 402 (2011). arXiv: 1106.0715
39. A.J.M. Medved, E.C. Vagenas, Phys. Rev. D 70, 124021 (2004). arXiv:hep-th/0411022

40. R.A. El-Nabulsi, Quantum Stud. Math. Found. 6, 235 (2019). https://doi.org/10.1007/s40509-019-00181-x

41. C. Adami, arXiv:quant-ph/0405005

42. P. Bargueño, E. C. Vagenas, Phys. Lett. B 742, 15 (2015). arXiv: 1501.03256

43. N. M.-Durán, A.F. Vargas, P. Hoyos-Restrepo, P. Barguen̄o, Eur. Phys. J. C 76, 559 (2016). arXiv:1606.06635

44. Z.-W. Feng, S.-Z. Yang, Phys. Lett. B 772, 737 (2017). arXiv: 1501.03256

45. Z.F. Gao, X.-D. Li, N. Wang, J.P. Yuan, P. Wang, Q.H. Peng, Y.J. Du, Mon. Not. R. Astron. Soc. 456, 55 (2016). https://doi.org/10. 1093/mnras/stv2465

46. Z.F. Gao, D.L. Song, X.D. Li, H. Shan, N. Wang, Astron. Nachr. 340, 241 (2019). https://doi.org/10.1002/asna.201913599

47. Z.-Y. Fu, H.-L. Li, Y. Li, D.-W. Song, Eur. Phys. J. Plus 135, 125 (2020). https://doi.org/10.1140/epjp/s13360-020-00190-5

48. F. Scardigli, Symmetry 12, 1519 (2020). https://doi.org/10.1002/ asna.201913599, https://doi.org/10.3390/sym12091519

49. A. Iorio, G. Lambiase, P. Pais, F. Scardigli, Phys. Rev. D 101, 105002 (2020). arXiv: 1910.09019

50. F. Scardigli, Class. Quantum Gravity 14, 1781 (1997). arXiv:gr-qc/9706030

51. I. Sakalli, Int. J. Theor. Phys. 50, 2426 (2011). arXiv: 1103.1728

52. A. Sheykhi, H. Moradpour, N. Riazi, Gen. Relativ. Gravit. 45, 1033 (2013). arXiv:1109.3631

53. B. Hamil, B.C. Lütfüoğlu, arXiv:2009.13838

54. R.A. El-Nabulsi, Eur. Phys. J. Plus 135, 34 (2020). https://doi.org/ 10.1140/epjp/s13360-019-00051-w

55. S. Das, E.C. Vagenas, Phys. Rev. Lett. 101, 221301 (2008). https:// doi.org/10.1103/PhysRevLett.101.221301

56. F. Marin, F. Marino, M. Bonaldi, M. Cerdonio, L. Conti, P. Falferi, R. Mezzena, A. Ortolan, G.A. Prodi, L. Taffarello, G. Vedovato, A. Vinante, J.P. Zendri, Nat. Phys. 9, 71 (2013). https://doi.org/10. $1038 /$ nphys 2503

57. S. Ghosh, Class. Quantum Gravity 31, 025025 (2014). arXiv: 1303.1256

58. F. Scardigli, R. Casadio, Eur. Phys. J. C 75, 425 (2015). arXiv: 1407.0113

59. D. Gao, M. Zhan, Phys. Rev. A 94, 013607 (2016). arXiv:1607.04353

60. F. Scardigli, G. Lambiase, E.C. Vagenas, Phys. Lett. B 767, 242 (2017). arXiv:1709.00637

61. F. Scardigli, J. Phys. Conf. Ser. 1275, 012004 (2019) arXiv: 1905.00287

62. A.F. Ali, S. Das, E.C. Vagenas, Phys. Rev. D 84, 044013 (2011). arXiv: 1107.3164

63. P. Bushev, J. Bourhill, M. Goryachev, N. Kukharchyk, E. Ivanov, S. Galliou, M. Tobar, S. Danilishin, Phys. Rev. D 100, 066020 (2019). arXiv: 1903.03346

64. Z.-W. Feng, S.-Z. Yang, H.-L. Li, X.-T. Zu, Phys. Lett. B 768, 81 (2017). arXiv: 1610.08549

65. S. Kouwn, Phys. Dark Universe 21, 76 (2018). arXiv:1805.07278

66. S. Giardino, V. Salzano, arXiv:2006.01580

67. J.C.S. Neves, Eur. Phys. J. C 80, 343 (2020). arXiv:1906.11735

68. S. Das, R. Mann, Phys. Lett. B 704, 596 (2011). arXiv:1109.3258

69. S. Bhattacharyya, S. Gangopadhyay, A. Saha, Class. Quantum Gravity 37, 195006 (2020). https://doi.org/10.1088/1361-6382/ abac45

70. Ö. Ökcü, E. Aydiner, Nucl. Phys. B 964, 115324 (2021). arXiv:2101.09524

71. A. Das, S. Das, N.R. Mansour, E.C. Vagenas, Phys. Lett. B 819, 136429 (2021). arXiv:2101.03746 